\title{
Impact of Dysnatremia and Dyskalemia on Prognosis in Patients with Aneurysmal Subarachnoid Hemorrhage: A Retrospective Study
}

\author{
Catherine WY Tam ${ }^{1}$, HP Shum², WW Yan ${ }^{3}$
}

\begin{abstract}
Background: Electrolyte disturbance is one of the complications of subarachnoid hemorrhage (SAH) and its prognostic value is not fully understood. The focus of this study is to evaluate the impact of dysnatremia and dyskalemia on functional outcomes in patients with aneurysmal $\mathrm{SAH}$.

Materials and methods: Patients with spontaneous aneurysmal SAH who were admitted to our intensive care unit (ICU) between 1 st January 2011 and 31st December 2016 were included. Demographic data, biochemical parameters from days 1 to day 11 of ICU admission, disease severity, and clinical outcome were recorded. The prognosis was estimated using the Glasgow outcome scale (GOS) at 3 months after the initial insult. Results: A total of 244 patients were included in this study. There were 139 patients (57.0\%) with hyponatremia ( $\mathrm{Na}<135 \mathrm{mmol} / \mathrm{L})$ while 82 patients ( $33.6 \%$ ) had hypernatremia ( $\mathrm{Na}>146 \mathrm{mmol} / \mathrm{L}$ ). Hyponatremia, hypernatremia, and sodium fluctuation $>12 \mathrm{mmol} / \mathrm{L}$ were $\mathrm{more}$ commonly found in those patients with poor outcome. However, both hypokalemia and hyperkalemia were not shown to have a significant effect on the patient's prognosis. Logistic regression analysis identified the following independent predictors of poor outcome (GOS 1-3 at 3 months): age $>55$ years old, acute physiology and chronic health evaluation IV (APACHE IV) score $>50$, World Federation of Neurosurgical Societies (WFNS) grade $>3$, Fisher grade $>2$, presence of intracranial hemorrhage (ICH)/intraventricular hemorrhage (IVH), use of mannitol, use of loop diuretic aneurysms, involving posterior circulation, and hypernatremia $>146 \mathrm{mmol} / \mathrm{L}$.

Conclusion: Hypernatremia, but not hyponatremia, in patients with aneurysmal SAH is associated with poor outcome. Both hypokalemia and hyperkalemia were not shown to have a significant effect on the patient's prognosis. Further studies are required to determine whether the treatment of dysnatremia can influence outcomes.

Clinical significance: Dysnatremia and dyskalemia are common in patients with aneurysmal SAH, but only hypernatremia is associated with poor outcome. Further studies are required to determine whether the treatment of dysnatremia can influence outcomes.

Keywords: Clinical outcome, Hypernatremia, Hyponatremia, Subarachnoid hemorrhage.

Indian Journal of Critical Care Medicine (2019): 10.5005/jp-journals-10071-23292
\end{abstract}

\section{INTRODUCTION}

Aneurysmal SAH itself carries high disability and mortality rates. ${ }^{1}$ The incidence of SAH has been estimated to be approximately 9 per $1,00,000$ person-years, with geographic variations and association with age. ${ }^{2}$ In our locality, the incidence of SAH was 7.5 per 1,00,000 person-years in 2010 and that the trend was increasing over the following years. ${ }^{3}$ Although the main predictive factor of $\mathrm{SAH}$ outcome is the severity of neurological morbidity, non-neurological complications can also affect the ICU, and hospital length of stay (LOS). ${ }^{4}$ Electrolyte disturbance, especially both hyponatremia and hypernatremia, are frequently observed in the acute phase of SAH. ${ }^{5}$ The management of hyponatremia is often a challenge because of the varying etiologies and treatment direction. The outcomes associated with altered serum sodium levels in SAH patients are not fully understood. Some studies have shown conflicting results regarding the relationship between altered serum sodium levels and prognosis. ${ }^{1,6,7}$ The objective of this study is to evaluate the impact of dysnatremia and dyskalemia on prognostic functional outcomes in aneurysmal SAH patients.

\section{Materials and Methods}

This is a single-center, retrospective cohort study conducted in the ICU of Pamela Youde Nethersole Eastern Hospital (PYNEH), which is a tertiary regional hospital in Hong Kong. Around $95 \%$ of all $\mathrm{SAH}$
${ }^{1}$ Department of Radiology, North District Hospital, Sheung Shui, Hong Kong SAR, China

${ }^{2,3}$ Department of Intensive Care, Pamela Youde Nethersole Eastern Hospital, Chai Wan, Hong Kong SAR, China

Corresponding Author: HP Shum, Department of Intensive Care, Pamela Youde Nethersole Eastern Hospital, Chai Wan, Hong Kong SAR, China, Phone: +852 853-97337363, e-mail: shumhp@ha.org.hk

How to cite this article: Tam CWY, Shum HP, Yan WW. Impact of Dysnatremia and Dyskalemia on Prognosis in Patients with Aneurysmal Subarachnoid Hemorrhage: A Retrospective Study. Indian J Crit Care Med 2019;23(12):562-567.

Source of support: Nil

Conflict of interest: None to declare for all authors. Part of this work has been presented in poster format at the 38th International Symposium on Intensive Care and Emergency Medicine.

admissions to our hospital were treated in our ICU. This study was approved by the Hong Kong East Cluster Ethics Committee. Written informed consent was waived due to the retrospective, anonymous nature of the analysis.

\section{Study Population}

This study included all patients with spontaneous aneurysmal SAH who were admitted to our ICU between January 1, 2011 and

o The Author(s). 2019 Open Access This article is distributed under the terms of the Creative Commons Attribution 4.0 International License (https://creativecommons. org/licenses/by-nc/4.0/), which permits unrestricted use, distribution, and non-commercial reproduction in any medium, provided you give appropriate credit to the original author(s) and the source, provide a link to the Creative Commons license, and indicate if changes were made. The Creative Commons Public Domain Dedication waiver (http://creativecommons.org/publicdomain/zero/1.0/) applies to the data made available in this article, unless otherwise stated. 
Impact of Dysnatremia and Dyskalemia on Prognosis in Patients with Aneurysmal Subarachnoid Hemorrhage

December 31, 2016. Both elective and emergency admissions were included in this study. Individuals were at least 18 years of age and exhibited spontaneous SAH (as diagnosed with computed tomography) with a confirmed aneurysm on angiography were included. Patients with high suspicion of aneurysmal SAH based on history or confirmed SAH on computerized tomography (CT) scan, however, with high-grade $\mathrm{SAH}$ on presentation deemed not suitable for further intervention or angiography were also included. Patients with traumatic SAH and those with missing data were excluded.

\section{Data Collection}

Patient data were collected from paper and electronic medical records. Collected data included demographic details, comorbidities, clinical characteristics (APACHE IV score), laboratory parameters, presence of vasospasm, severity of SAH based on clinical grading (WFNS grade), and radiological scaling (Fisher grade), location and size of aneurysm, and treatment modality. Outcome data included ICU/hospital LOS and discharge conditions were extracted. For recurrent ICU admissions during the same hospitalization episode, only the first ICU admission episode was included.

\section{Definitions}

\section{Electrolytes Disturbance}

Serum sodium levels were retrieved daily on admission to ICU (day 1 ) up to day 11 after SAH. Hyponatremia was defined by a serum sodium level $<135 \mathrm{mmol} / \mathrm{L}$ while hypernatremia was defined by a serum sodium level of $>146 \mathrm{mmol} / \mathrm{L}$. If levels of sodium and potassium were checked more than once per day, the minimum and maximum levels for both electrolytes between 00:00 and 23:59 of day 1 were recorded. Fluctuation values for sodium were obtained by subtracting the minimum from maximum sodium levels from the date of admission to death or day 11 whichever earlier and were categorized as $<12 \mathrm{mmol} / \mathrm{L}$ or $>12 \mathrm{mmol} / \mathrm{L}$. ${ }^{7}$ Hypokalemia was defined by a serum potassium level $<3 \mathrm{mmol} / \mathrm{L}$ while hyperkalemia was defined by a serum potassium level $>5 \mathrm{mmol} / \mathrm{L}$.

\section{Grading of Subarachnoid Hemorrhage}

The clinical grading (WFNS grade) of the patients and the radiological scaling (Fisher grade) were determined by the revision of all CT films and clinical parameters. ${ }^{8,9}$

\section{Cerebral Vasospasm}

Cerebral vasospasm was determined by the transcranial Doppler (TCD) evaluation. One nurse specialist performed all the scanning procedure. Four main acoustic windows (transtemporal window, transorbital window, submandibular window, and suboccipital window) were used to identify the measurements of blood flow at each major branch of the circle of Willis. ${ }^{10}$ Mean flow velocities of $>100 \mathrm{~cm} /$ seconds were considered as vasospasm. ${ }^{11}$ For those with difficult TCD assessment, CT angiography, or digital subtraction angiography was used for diagnosis of cerebral vasospasm.

\section{Patient Care}

According to our standard operative procedures, patients with $\mathrm{SAH}$ were kept with a head-elevated position of $30^{\circ}-45^{\circ}$ with the head aligned to the body axis. Regular re-evaluation of neurologic status was performed by the intensivists and neurosurgeon. Continuous blood pressure monitoring was performed using an invasive arterial blood pressure monitoring device. The initial target systolic blood pressure was $\leq 160 \mathrm{~mm} \mathrm{Hg}$ with cerebral perfusion pressure of $>60 \mathrm{~mm} \mathrm{Hg}$. Candidacy for endovascular interventional radiologic (IR) vs surgical obliteration of the aneurysm is evaluated by an experienced team of neurosurgeons and neuroradiologists at presentation and treatment modality is up to their discretion. Nimodipine, which may reduce vasospasm and reduce the risk of secondary cerebral ischemia, was routinely prescribed. Medical treatment of electrolyte disturbance in ICU is provided at the discretion of the intensivists. All patients with documented cerebral vasospasm were treated with induced hypertension using noradrenaline with a target mean arterial pressure of $90-110 \mathrm{~mm} \mathrm{Hg}$ and volume optimization using isotonic crystalloid solution targeting euvolemia. Endovascular therapies including intra-arterial vasodilator therapy (using verapamil) and balloon angioplasty were at the discretion of neuroradiologists and neurosurgeons for management of severe cerebral vasospasm. Central venous pressures were not routinely measured.

\section{Outcome}

The primary outcome was 3-month Glasgow outcome score (GOS), which was calculated from the first date of admission to ICU due to SAH (Table 1). ${ }^{12}$ The GOS score 1-3 is considered as poor outcome, whereas the GOS score $4-5$ is a good outcome. Secondary outcomes were ICU and hospital mortality, ICU and hospital LOS.

\section{Statistical Analyses}

The estimated SAH incidence in our locality is 7.5 per 100,000 populations. ${ }^{3}$ With a $95 \%$ confidence interval and a $10 \%$ margin of error, the calculated sample size for this study is 94 in order to provide representative findings.

For the data analysis, categorical variables were compared using Pearson Chi-square tests or Fisher's exact test as appropriate. Student $t$ test or Mann-Whitney $U$ test was used to compare continuous variables. Pearson's and Spearman test were used to determine the correlation between vasospasm and serum sodium level. Results were expressed as mean \pm standard deviation or as number of cases and percentage as appropriate. Logistic regression analysis with forward stepwise approach (instead of Cox regression analysis) was used to assess independent predictors for poor outcome (3-month GOS 1-3) because the time for when GOS 4 transits to 3 could not be clearly defined. Factors within the logistic regression model included the following: age more than 55 ; APACHE IV score $>50$; gender; WFNS grade $>3$; Fisher grade $>2$;

Table 1: Glasgow outcome score

\begin{tabular}{ll}
\hline Score & Description \\
\hline 1 & Death \\
2 & $\begin{array}{l}\text { Persistent vegetative state } \\
\text { Patient exhibits no obvious cortical function }\end{array}$ \\
3 & Severe disability \\
& Conscious but disabled. Patient depends on others for \\
daily support & Moderate disability \\
4 & $\begin{array}{l}\text { Disabled but independent. Patient is independent as far as } \\
\text { daily life. Disabilities include varying degrees of dysphasia, } \\
\text { hemiparesis, or ataxia as well as intellectual and memory } \\
\text { deficits and personality changes } \\
\text { Good recovery/low disability } \\
\text { Resumption of normal activities even though there may be } \\
\text { minor neurologic or psychological deficits }\end{array}$ \\
&
\end{tabular}


presence of $\mathrm{ICH} / \mathrm{IVH}$; received IR procedures; use of mannitol or loop diuretic; aneurysm involving posterior circulation; day 1-11 sodium $<135 \mathrm{mmol} / \mathrm{L}$; day $1-11$ sodium $>146 \mathrm{mmol} / \mathrm{L}$; day $1-11$ sodium variability (max-min sodium level $>12 \mathrm{mmol} / \mathrm{L}$ ); and day 1-11 potassium $>5 \mathrm{mmol} / \mathrm{L}$. The factor selection for the regression model was based on $p<0.1$ within the univariate analyses, and avoidance of collinearity. Regression model fit was assessed using the Hosmer and Lemeshow goodness-of-fit test, Cox and Snell $R$ square test and Nagelkerke $R$ square test. Continuous variables were converted to categorical variables with appropriate cutoff values determined by receiver operating characteristic curve analysis. Statistical analysis was done using the Statistical Package for Social Sciences version 20 (SPSS Inc., Chicago, IL). $p$ values $<0.05$ were considered statistically significant for this study.

\section{Results}

\section{All Patients}

A total of 252 SAH patients were admitted to our ICU from January 2011 to December 2016 and 244 patients were eligible for this study with eight patients excluded due to missing data $(n=4)$ and those with traumatic SAH $(n=4)$. The mean age of patients was $57.7 \pm 14.6$ years, $36.4 \%$ were men and the APACHE IV score was $59 \pm 31$ (Table 2). Concerning the severity of $\mathrm{SAH}, 43.9 \%$ had WFNS grade $>3,75 \%$ had Fisher grade $>2$, and $63.5 \%$ associated with $\mathrm{ICH} / \mathrm{IVH}$. Aneurysms were commonly located at the anterior circulation (62.7\%). Around half of the patients (55.7\%) received radiologic interventions and only $21.7 \%$ were treated with surgical clipping. Documented vasospasm was reported in $41.4 \%$ of patients. Hyponatremia ( $\mathrm{Na}<135 \mathrm{mmol} / \mathrm{L}$ ) occurred in $57 \%$ of patients while hypernatremia ( $\mathrm{Na}>146 \mathrm{mmol} / \mathrm{L}$ ) occurred in $33.6 \%$ of patients. Sodium level fluctuation $>12 \mathrm{mmol} / \mathrm{L}$ was noted in $39.3 \%$ of patients. Hypokalemia ( $<3 \mathrm{mmol} / \mathrm{L}$ ) and hyperkalemia ( $>5 \mathrm{mmol} / \mathrm{L}$ ) occurred in 29.5 and $21.3 \%$ of patients. The average ICU and hospital LOS were $7.6 \pm 6.2$ and $21.1 \pm 27.5$ days, respectively. The ICU, hospital and 3 -month mortality were $18,24.2$, and $25.4 \%$, respectively.

\section{Comparison between those with Poor (GOS 1-3) and Favorable Outcome (GOS 4-5)}

Among 244 recruited patients, half of them (122) had a poor outcome while half of them (122) had a favorable outcome (Table 2). Patients with poor outcomes were older, more likely to be male gender, had lower Glasgow coma scale (GCS) on ICU day 1 and higher disease severity as evidenced by higher APACHE score, higher WFNS grade, and Fisher grade. The presence of posterior circulation locating aneurysm, coexisting $\mathrm{ICH} / \mathrm{IVH}$ and the use of mannitol or loop diuretics pointed to a poor outcome. Comparing treatment modality, patients who underwent IR procedures had a better outcome. Hyponatremia, hypernatremia, and sodium fluctuation $>12 \mathrm{mmol} / \mathrm{L}$ were more commonly found in those patients with poor outcome. However, both hypokalemia and hyperkalemia were not shown to have a significant effect on

Table 2: Comparison between those with Glasgow outcome scale (GOS) 1-3 vs those with GOS 4-5

\begin{tabular}{|c|c|c|c|c|}
\hline Parameters & All patients $(n=244)$ & GOS $4-5(n=122)$ & $\operatorname{GOS} 1-3(n=122)$ & $p$ value \\
\hline Age (years) & $57.7 \pm 14.6$ & $52.7 \pm 13.9$ & $62.8 \pm 13.6$ & $<0.001$ \\
\hline Male & $89(36.4)$ & $36(30.0)$ & $53(43.4)$ & 0.024 \\
\hline \multicolumn{5}{|l|}{ Source of admission } \\
\hline General ward & $38(15.6)$ & $20(16.4)$ & $18(14.8)$ & 0.113 \\
\hline OR/recovery & $181(74.2)$ & $94(77.0)$ & $87(71.3)$ & \\
\hline Others & $25(10.2)$ & $8(6.6)$ & $17(13.9)$ & \\
\hline APACHE IV score & $59 \pm 31$ & $42 \pm 22$ & $77 \pm 28$ & $<0.001$ \\
\hline APACHE IV risk of death & $0.25 \pm 0.24$ & $0.12 \pm 0.14$ & $0.37 \pm 0.26$ & $<0.001$ \\
\hline Day 1 lowest GCS & $8 \pm 5$ & $11 \pm 4$ & $6 \pm 4$ & $<0.001$ \\
\hline \multicolumn{5}{|l|}{ WFNS grade } \\
\hline 1 & $97(39.8)$ & $77(63.1)$ & $20(16.4)$ & $<0.001$ \\
\hline 2 & $22(9.0)$ & $11(9.0)$ & $11(9.0)$ & \\
\hline 3 & $18(7.4)$ & $11(9.0)$ & $7(5.7)$ & \\
\hline 4 & $40(16.4)$ & $12(9.8)$ & $28(23.0)$ & \\
\hline 5 & $67(27.5)$ & $11(9.0)$ & $56(45.9)$ & \\
\hline \multicolumn{5}{|l|}{ Fisher grade } \\
\hline 1 & $35(14.3)$ & $31(25.4)$ & $4(3.2)$ & $<0.001$ \\
\hline 2 & $26(10.7)$ & $17(13.9)$ & $9(7.4)$ & \\
\hline 3 & $54(22.1)$ & $43(35.2)$ & $11(9.0)$ & \\
\hline 4 & $129(52.9)$ & $31(25.4)$ & $98(80.3)$ & \\
\hline \multicolumn{5}{|l|}{ Aneurysm location } \\
\hline Anterior circulation & $153(62.7)$ & $90(73.8)$ & $63(51.6)$ & 0.002 \\
\hline Posterior circulation & $66(27.0)$ & $24(19.7)$ & $42(34.4)$ & \\
\hline Not clear & $25(10.3)$ & $8(6.6)$ & $17(13.9)$ & \\
\hline Aneurysm maximum size (mm) & $5.6 \pm 5.8$ & $5.4 \pm 3.7$ & $5.7 \pm 7.3$ & 0.658 \\
\hline Presence of $\mathrm{ICH} / \mathrm{IVH}$ & $155(63.5)$ & $48(39.3)$ & $107(87.7)$ & $<0.001$ \\
\hline \multirow[t]{2}{*}{ Presence of vasospasm } & $101(41.4)$ & $45(36.9)$ & $56(45.9)$ & 0.153 \\
\hline & & & & Contd \\
\hline Indian Journal of Critical Care & , Volume 23 Issue 12 (De & 2019) & & \\
\hline
\end{tabular}


Contd...

\begin{tabular}{|c|c|c|c|c|}
\hline Parameters & All patients $(n=244)$ & GOS $4-5(n=122)$ & $\operatorname{GOS} 1-3(n=122)$ & pvalue \\
\hline \multicolumn{5}{|l|}{ Interventions } \\
\hline Interventional radiological procedures & $136(55.7)$ & $87(71.3)$ & $49(40.2)$ & $<0.001$ \\
\hline Surgical clipping & $53(21.7)$ & $31(25.4)$ & $22(18.0)$ & 0.162 \\
\hline \multicolumn{5}{|l|}{ Medications } \\
\hline Mannitol & $33(13.5)$ & $8(6.6)$ & $25(20.5)$ & 0.001 \\
\hline Loop diuretics & $44(18.0)$ & $10(8.2)$ & $34(27.9)$ & $<0.001$ \\
\hline \multicolumn{5}{|l|}{ Sodium (mmol/L) } \\
\hline Days $1-11$ minimum sodium & $133 \pm 4$ & $132 \pm 4$ & $135 \pm 4$ & $<0.001$ \\
\hline Days $1-11$ maximum sodium & $145 \pm 6$ & $143 \pm 5$ & $148 \pm 7$ & $<0.001$ \\
\hline Days $1-11$ hyponatremia $(<135)$ & $139(57.0)$ & $86(70.5)$ & $53(43.4)$ & $<0.001$ \\
\hline Days 1-11 hypernatremia (>146) & $82(33.6)$ & $16(13.1)$ & $66(54.1)$ & $<0.001$ \\
\hline Days $1-11$ sodium change $>12$ & $96(39.3)$ & $33(27.0)$ & $63(51.6)$ & $<0.001$ \\
\hline \multicolumn{5}{|l|}{ Potassium (mmol/L) } \\
\hline Days $1-11$ minimum potassium & $3.1 \pm 0.3$ & $3.1 \pm 0.3$ & $3.1 \pm 0.4$ & 0.409 \\
\hline Days 1-11 maximum potassium & $4.6 \pm 0.6$ & $4.5 \pm 0.6$ & $4.7 \pm 0.7$ & 0.006 \\
\hline Days $1-11$ hypokalemia $(<3)$ & $72(29.5)$ & $38(31.1)$ & $34(27.9)$ & 0.574 \\
\hline Days $1-11$ hyperkalemia $(>5)$ & $52(21.3)$ & $20(16.4)$ & $32(26.2)$ & 0.061 \\
\hline \multicolumn{5}{|l|}{ Length of stay (days) } \\
\hline ICU & $7.6 \pm 6.2$ & $6.8 \pm 5.7$ & $8.5 \pm 6.6$ & 0.036 \\
\hline Hospital & $21.1 \pm 27.5$ & $16.5 \pm 9.2$ & $25.6 \pm 37.3$ & 0.009 \\
\hline \multicolumn{5}{|l|}{ Mortality } \\
\hline ICU & $44(18.0)$ & $0(0)$ & $44(36.1)$ & $<0.001$ \\
\hline Hospital & $59(24.2)$ & $0(0)$ & $59(48.0)$ & $<0.001$ \\
\hline 30-day & $57(23.4)$ & $0(0)$ & $57(46.7)$ & $<0.001$ \\
\hline 3-month & $62(25.4)$ & $0(0)$ & $62(50.8)$ & $<0.001$ \\
\hline
\end{tabular}

All data area show as mean \pm standard deviation or number (\%) unless otherwise specified

patient's prognosis. The ICU and hospital LOS were longer for those with poor outcome.

\section{Independent Predictors of Poor Outcome}

Logistic regression analysis using a forward stepwise approach identified the following independent predictors for poor outcome (GOS 1-3) at 3 months (Table 3): age $\geq 55$ years old, APACHE IV score $>50$, WFNS grade $>3$, Fisher grade $>2$, the presence of $\mathrm{ICH} / \mathrm{IVH}$, use of mannitol, use of loop diuretic, aneurysm involving the posterior circulation, and hypernatremia ( $>146 \mathrm{mmol} / \mathrm{L}$ ). Patients who received IR procedure had a better outcome (GOS $4-5$ at 3 months).

\section{Discussion}

This study showed that days 1-11 hypernatremia (sodium $>146$ $\mathrm{mmol} / \mathrm{L}$ ), but not hyponatremia independently predicted poor outcome at 3 months for those with aneurysmal SAH. Other poor outcome predictors included: age $>55$, APACHE IV score $>50$, WFNS grade $>3$, Fisher grade $>2$, presence of $\mathrm{ICH} / \mathrm{IVH}$, use of mannitol or loop diuretics, and aneurysm involving posterior circulation. Those who received IR procedure had a better outcome.

The majority of non-traumatic SAH cases observed in our study are due to ruptured intracranial aneurysm (93\%), similar to another study. ${ }^{13} \mathrm{~A}$ higher proportion of our patient population is women (63.6\%) and $15 \%$ of our patients have multiple aneurysms which correlate with the literature. ${ }^{14}$ The median age on SAH presentation was 50 years old while the mean age at aneurysmal rupture was 55 years old. ${ }^{15}$ We observed that there was a worsening of the outcome
Table 3: Logistic regression analysis to identify independent predictors for poor outcome (3 months GOS 1-3) using forward stepwise approach

\begin{tabular}{lllr}
\hline & & \multicolumn{2}{l}{$95 \%$ confidence } \\
Parameters & Odds ratio & interval & p value \\
\hline Age $>55$ years old & 5.730 & $2.346-13.993$ & $<0.001$ \\
APACHE IV score $>50$ & 4.314 & $1.752-10.622$ & 0.001 \\
WFNS grade $>3$ & 2.747 & $1.067-7.072$ & 0.036 \\
Fisher grade $>2$ & 2.878 & $1.125-7.361$ & 0.027 \\
Presence of ICH/IVH & 4.055 & $1.603-10.259$ & 0.003 \\
Use of mannitol & 4.318 & $1.027-18.159$ & 0.046 \\
Use of loop diuretic & 6.022 & $1.994-18.189$ & 0.001 \\
Aneurysm involving & 3.882 & $1.435-10.499$ & 0.008 \\
posterior circulation & & & \\
Days 1-11 sodium & 3.003 & $1.166-7.732$ & 0.023 \\
$>146$ mmol/L & & & \\
Received IR procedures & 0.276 & $0.114-0.669$ & 0.004 \\
\hline
\end{tabular}

Hosmer and Lemeshow test $x^{2}=9.136, \mathrm{df}=8, p=0.331$ Cox and Snell $R$ square $52.1 \%$, Nagelkerke $R$ squared $69.5 \%$, correctly classified $86.5 \%$ of cases

when age on presentation was older than 55 years old. In fact, advanced age is a well-recognized prognostic indicator of poor outcome in patients with $\mathrm{SAH}$, which may be explained by poorer functional reserve and the presence of multiple comorbidities. ${ }^{1,16}$

Subarachnoid hemorrhage is associated with a high percentage of disability (33\%) and deaths (44\%). ${ }^{17}$ Our ICU mortality rate due 
Impact of Dysnatremia and Dyskalemia on Prognosis in Patients with Aneurysmal Subarachnoid Hemorrhage

to $\mathrm{SAH}$ was $18 \%$ and the in-hospital mortality rate is $24.2 \%$. Due to the diagnostics and therapeutic advancement for patients with $\mathrm{SAH}$, the prognosis for these patients has improved significantly in recent decades. ${ }^{18}$ However, similar to our study findings, poor WFNS score, and Fisher grade remain as independent predictors of poor outcome. ${ }^{1}$ A substantial amount of SAH can cause increased intracranial pressure and may decrease cerebral perfusion, which leads to increased cerebral edema and death. ${ }^{17}$

The presence of IVH/ICH in our study was also shown to be an independent predictor of poor outcome. Severity of aneurysmal IVH is a strong contributor to initial severity and early complications of $\mathrm{SAH}$, which leads to the poor outcome. ${ }^{19}$ Echoed with previous studies, aneurysms involving posterior circulation, but not the size of the aneurysm was shown to be associated with poorer outcome in our study. ${ }^{1,20}$ Differences in the types of neurological deficits due to posterior circulation involvement may explain this finding. ${ }^{21}$

In our study population, 57\% developed hyponatremia, which was similar to previous studies. ${ }^{22,23}$ The occurrence of hyponatremia was not associated with worsened neurological outcome at 3 months. The reason why hyponatremia did not lead to poor outcome can be attributed to cerebral autoregulation. It was discovered that cerebral tissue could adapt to hyponatremia and attain stable status several hours afterward. ${ }^{24}$ The prognostic significance of hyponatremia may be undermined in our study, possibly due to more aggressive fluid management and correction of hyponatremia. The significance in hyponatremia is less pronounced compared with hypernatremia in literature. Hypernatremia was significantly associated with poor outcome in our study and similar to previous studies. ${ }^{25,26}$ Although hypernatremia leads to increased cellular dehydration with decreased cerebral edema, which can be a therapeutic goal in those with poor-grade $\mathrm{SAH}$, this altered homeostatic state can lead to myelin damage and even neuronal death, which contributes to additional secondary brain injury after $\mathrm{SAH} .{ }^{25,26}$ Hypernatremia is also associated with the presence of acute kidney injury, which may contribute to the poor outcome. ${ }^{27}$ Our study showed that sodium variability $>12 \mathrm{mmol} / \mathrm{L}$ significantly worsened the outcome. Studies by Bales et al. and Engles et al. showed similar findings and fluctuations in serum sodium level contributed to the development of delayed cerebral ischemia in $\mathrm{SAH}$, resulting in guarded prognosis., ${ }^{7,28}$

One of the common causes of hypernatremia in neurosurgical patients is diabetes insipidus. A significant negative fluid balance status may indicate the presence of this complication. When compared with those patients without hypernatremia, patients with hypernatremia had higher (instead of lower) average fluid balance on the first 4 days of ICU admission $(368 \pm 622 \mathrm{~mL}$ vs $169 \pm 558 \mathrm{~mL}$, $p=0.012$ ). Given that $39.8 \%$ of the recruited patients had less than 4 days of ICU LOS and accurate fluid status information is difficult to obtain when the patients were discharged from the ICU, further analysis of the relationship of fluid balance and patient's outcome was not performed.

In our study, both hypokalemia and hyperkalemia had no significance on the outcome. Alimohamadis' prospective study showed that hypokalemia in the subacute phase of SAH (days 7-10) correlated with poor outcome while hyperkalemia was significantly associated with less radiographic severity. ${ }^{29}$ But the effect of potassium on outcome after $\mathrm{SAH}$ remains controversial. ${ }^{30} \mathrm{~A}$ retrospective observational cohort study showed that hypokalemia in patients with aneurysmal SAH in their acute phase is accompanied by elevated serum sodium level, which in terms contributes to poor outcome as mentioned before. ${ }^{31}$

There are several therapies aimed at reducing the intracranial pressure (ICP), including the use of osmotic diuretics with mannitol or hypertonic saline solution. Loop diuretic alone or in combination with mannitol can also be used to suppress ICP. A study showed that the combination resulted in a longer-lasting reduction in ICP than mannitol alone. ${ }^{32}$ We showed that the use of loop diuretic or mannitol independently predicted poor outcome, which yielded similar results as Sakr et al. ${ }^{33}$ The presence of high ICP and significant fluid overload, which drive the use of mannitol and loop diuretics may be the underlying reasons that contributed to a poor outcome.

We also found that patients who underwent IR treatment had a better prognosis. A systemic review showed that coiling is associated with a higher risk of rebleeding, but yields a better clinical outcome, especially in those patients with good preoperative status. ${ }^{34}$ In another retrospective study, clipping was shown to have a better outcome and lower mortality at discharge than coiling among good-grade patients. In patients with poorgrade, there was no difference in any outcome measure among the treatment groups. ${ }^{35}$ The definite choice of surgical intervention vs endovascular treatment, therefore, remains unclear.

\section{Limitations}

Our study has several limitations. First, a cause-effect relationship cannot be established with certainty because of the retrospective nature of the analysis and inherent limitations of the multivariable analysis. Second, the management of SAH may vary among institutions and geographic regions, which hinders the generalisability of our results. Third, serum sodium or potassium assays were not regularly performed for all patients, which may miss some critical values during ICU stay. Fourth, unreported confounding factors, e.g., neurosurgical techniques, operative conditions, and post-ICU discharge cares, might strongly affect patients' outcome. However, the single-center nature of the study may be an advantage as it limits the impact of variations in practice that can be a confounding factor in multicenter studies.

\section{Conclusion}

Hypernatremia, but not hyponatremia, in patients with aneurysmal $\mathrm{SAH}$ is associated with poor outcome. Both hypokalemia and hyperkalemia were not shown to have a significant effect on patient's prognosis. Further studies are required to determine whether the treatment of dysnatremia can influence outcomes.

\section{Clinical Significance}

Dysnatremia and dyskalemia are common in patients with aneurysmal SAH, but only hypernatremia is associated with poor outcome. Further studies are required to determine whether the treatment of dysnatremia can influence outcomes.

\section{Authors' Contributions}

HP Shum and Catherine WY Tam carried out primary literature search, study design, patient recruitment, data collection, statistical analysis, and drafted the manuscript. WW Yan carried out study design, initial drafting, and revised the manuscript. 


\section{References}

1. Rosengart AJ, Schultheiss KE, Tolentino J, Macdonald RL. Prognostic factors for outcome in patients with aneurysmal subarachnoid hemorrhage. Stroke 2007;38(8):2315-2321. DOI: 10.1161/STROKEAHA.107.484360.

2. de Rooij NK, Linn FH, van der Plas JA, Algra A, Rinkel GJ. Incidence of subarachnoid haemorrhage: a systematic review with emphasis on region, age, gender and time trends. J Neurol Neurosurg Psychiatry 2007;78(12):1365-1372. DOI: 10.1136/jnnp.2007.117655.

3. Wong GK, Wun Tam YY, Zhu XL, Poon WS. Incidence and mortality of spontaneous subarachnoid hemorrhage in Hong Kong from 2002 to 2010: a Hong Kong hospital authority clinical management system database analysis. World Neurosurg 2014;81(3-4):552-556. DOI: 10.1016/j.wneu.2013.07.128.

4. Naidech AM, Bendok BR, Tamul P, Bassin SL, Watts CM, Batjer HH, et al. Medical complications drive length of stay after brain hemorrhage: a cohort study. Neurocrit Care 2009;10(1):11-19. DOI: 10.1007/s12028008-9148-x.

5. Disney L, Weir B, Grace M, Roberts P.Trends in blood pressure, osmolality and electrolytes after subarachnoid hemorrhage from aneurysms. Can J Neurol Sci 1989;16(3):299-304. DOI: 10.1017/S0317167100029127.

6. McGirt MJ, Blessing R, Nimjee SM, Friedman AH, Alexander MJ, Laskowitz DT, et al. Correlation of serum brain natriuretic peptide with hyponatremia and delayed ischemic neurological deficits after subarachnoid hemorrhage. Neurosurgery 2004;54(6):1369-1373. DOI: 10.1227/01.NEU.0000125016.37332.50.

7. Bales J, Cho S, Tran TK, Korab GA, Khandelwal N, Spiekerman CF, et al. The effect of hyponatremia and sodium variability on outcomes in adults with aneurysmal subarachnoid hemorrhage. World Neurosurg 2016;96:340-349. DOI: 10.1016/j.wneu.2016.09.005.

8. Rosen DS, Macdonald RL. Subarachnoid hemorrhage grading scales: a systematic review. Neurocrit Care 2005;2(2):110-118. DOI: 10.1385/ NCC:2:2:110.

9. Hijdra A, Brouwers PJ, Vermeulen M, van Gijn J. Grading the amount of blood on computed tomograms after subarachnoid hemorrhage. Stroke 1990;21(8):1156-1161. DOI: 10.1161/01.STR.21.8.1156.

10. Purkayastha S, Sorond F. Transcranial Doppler ultrasound: technique and application. Semin Neurol 2012;32(4):411-420. DOI: 10.1055/s0032-1331812.

11. Compton JS, Redmond S, Symon L. Cerebral blood velocity in subarachnoid haemorrhage: a transcranial Doppler study. J Neurol Neurosurg Psychiatry 1987;50(11):1499-1503.DOI: 10.1136/jnnp.50.11.1499.

12. Jennett $B$, Bond $M$. Assessment of outcome after severe brain damage. Lancet 1975;1(7905):480-484. DOI: 10.1016/S0140-6736(75)92830-5.

13. van Gijn J, Rinkel GJ. Subarachnoid haemorrhage: diagnosis, causes and management. Brain 2001;124(Pt 2):249-278. DOI: 10.1093/ brain/124.2.249.

14. Kaminogo M, Yonekura M, Shibata S. Incidence and outcome of multiple intracranial aneurysms in a defined population. Stroke 2003;34(1):16-21. DOI: 10.1161/01.STR.0000046763.48330.AD.

15. Mayberg MR, Batjer HH, Dacey R, Diringer M, Haley EC, Heros RC, et al. Guidelines for the management of aneurysmal subarachnoid hemorrhage. A statement for healthcare professionals from a special writing group of the Stroke Council, American Heart Association. Circulation 1994;90(5):2592-2605. DOI: 10.1161/01.CIR.90.5.2592.

16. Lanzino G, Kassell NF, Germanson TP, Kongable GL, Truskowski LL, Torner JC, et al. Age and outcome after aneurysmal subarachnoid hemorrhage: why do older patients fare worse? J Neurosurg 1996;85(3):410-418. DOI: 10.3171/jns.1996.85.3.0410.

17. Claassen J, Carhuapoma JR, Kreiter KT, Du EY, Connolly ES, Mayer SA. Global cerebral edema after subarachnoid hemorrhage: frequency, predictors, and impact on outcome. Stroke 2002;33(5):1225-1232. DOI: 10.1161/01.STR.0000015624.29071.1F.

18. Lovelock CE, Rinkel GJ, Rothwell PM. Time trends in outcome of subarachnoid hemorrhage: population-based study and systematic review. Neurology 2010;74(19):1494-1501. DOI: 10.1212/ WNL.0b013e3181dd42b3.
19. Darkwah Oppong $M$, Gembruch O, Herten A, Frantsev R, Chihi $M$, Dammann $P$, et al. Intraventricular hemorrhage caused by subarachnoid hemorrhage: does the severity matter? World Neurosurg 2018;111:e693-e702. DOI: 10.1016/j.wneu.2017.12.148.

20. Nieuwkamp DJ, Setz LE, Algra A, Linn FH, de Rooij NK, Rinkel GJ. Changes in case fatality of aneurysmal subarachnoid haemorrhage over time, according to age, sex, and region: a meta-analysis. Lancet Neurol 2009;8(7):635-642. DOI: 10.1016/S1474-4422(09)70126-7.

21. Kim JT, Park MS, Choi KH, Kim BJ, Han MK, Park TH, et al. Clinical outcomes of posterior versus anterior circulation infarction with low National Institutes of Health Stroke Scale Scores. Stroke 2017;48(1): 55-62. DOI: 10.1161/STROKEAHA.116.013432.

22. Wijdicks EF, Vermeulen M, Hijdra A, van Gijn J. Hyponatremia and cerebral infarction in patients with ruptured intracranial aneurysms: is fluid restriction harmful? Ann Neurol 1985;17(2):137-140.DOI: 10.1002/ ana.410170206.

23. Sherlock M, O'Sullivan E, Agha A, Behan LA, Rawluk D, Brennan P, et al. The incidence and pathophysiology of hyponatraemia after subarachnoid haemorrhage. Clin Endocrinol 2006;64(3):250-254. DOI: 10.1111/j.1365-2265.2006.02432.x.

24. Gullans SR, Verbalis JG. Control of brain volume during hyperosmolar and hypoosmolar conditions. Annu Rev Med 1993;44:289-301. DOI: 10.1146/annurev.me.44.020193.001445.

25. Takaku A, Shindo K, Tanaka S, Mori T, Suzuki J. Fluid and electrolyte disturbances in patients with intracranial aneurysms. Surg Neurol 1979;11(5):349-356.

26. Beseoglu K, Etminan N, Steiger HJ, Hanggi D. The relation of early hypernatremia with clinical outcome in patients suffering from aneurysmal subarachnoid hemorrhage. Clin Neurol Neurosurg 2014;123:164-168. DOI: 10.1016/j.clineuro.2014.05.022.

27. Kumar AB, Shi Y, Shotwell MS, Richards J, Ehrenfeld JM. Hypernatremia is a significant risk factor for acute kidney injury after subarachnoid hemorrhage: a retrospective analysis. Neurocrit Care 2015;22(2): 184-191. DOI: 10.1007/s12028-014-0067-8.

28. Eagles ME, Tso MK, Macdonald RL. Significance of fluctuations in serum sodium levels following aneurysmal subarachnoid hemorrhage: an exploratory analysis. J Neurosurg 2018;131(2): 420-425. DOI: 10.3171/2018.3.JNS173068.

29. Alimohamadi M, Saghafinia M, Alikhani F, Danial Z, Shirani M, Amirjamshidi A. Impact of electrolyte imbalances on the outcome of aneurysmal subarachnoid hemorrhage: a prospective study. Asian J Neurosurg 2016;11(1):29-33. DOI: 10.4103/1793-5482.154978.

30. Kamp MA, Dibue M, Schneider T, Steiger HJ, Hanggi D. Calcium and potassium channels in experimental subarachnoid hemorrhage and transient global ischemia. Stroke Res Treat 2012;2012:382146. DOI: 10.1155/2012/382146.

31. Kimura H, Akutsu N, Shiomi R, Kohmura E. Subarachnoid hemorrhage caused by ruptured intracranial fusiform aneurysm associated with microscopic polyangiitis. Neurol Med Chir 2012;52(7):495-498. DOI: 10.2176/nmc.52.495.

32. Roberts PA, Pollay M, Engles C, Pendleton B, Reynolds E, Stevens FA. Effect on intracranial pressure of furosemide combined with varying doses and administration rates of mannitol. J Neurosurg 1987;66(3):440-446. DOI: 10.3171/jns.1987.66.3.0440.

33. Sakr Y, Dunisch P, Santos C, Matthes L, Zeidan M, Reinhart K, et al. Poor outcome is associated with less negative fluid balance in patients with aneurysmal subarachnoid hemorrhage treated with prophylactic vasopressor-induced hypertension. Ann Intensive Care 2016;6(1):25. DOI: 10.1186/s13613-016-0128-6.

34. Li H, Pan R, Wang H, Rong X, Yin Z, Milgrom DP, et al. Clipping versus coiling for ruptured intracranial aneurysms: a systematic review and meta-analysis. Stroke 2013;44(1):29-37. DOI: 10.1161/ STROKEAHA.112.663559.

35. Hoh BL, Topcuoglu MA, Singhal AB, Pryor JC, Rabinov JD, Rordorf $\mathrm{GA}$, et al. Effect of clipping, craniotomy, or intravascular coiling on cerebral vasospasm and patient outcome after aneurysmal subarachnoid hemorrhage. Neurosurgery 2004;55(4):779-786. DOI: 10.1227/01.NEU.0000137628.51839.D5. 